TecnoHumanismo. Revista Científica

Setiembre - Noviembre 2021

Volumen 1 / No.3

ISSN: $2710-2394$

pp. 488-509

https://doi.org/10.53673/th.v2i2.111



\title{
Formación y desarrollo profesional del contador público para el ejercicio de la asesoría financiera
}

Training and professional development of the public accountant for the exercise of financial advice Formação e desenvolvimento profissional do contador público para o exercício da assessoria financeira

\section{ARTÍCULO GENERAL}

\author{
Sinforiano Martínez-Huisa \\ smartinezhuisa@gmail.com \\ https://orcid.org/0000-0002-6933-1137
}

Universidad Nacional Federico Villareal, Lima - Perú

\author{
Domingo Hernández-Celis \\ dr.domingohernandezcelis@gmail.com \\ https://orcid.org/0000-0002-9759-4436
}

Universidad Nacional Federico Villareal, Lima - Perú

Recibido 07 de Abril 2021 | Arbitrado y aceptado 19 de Junio 2021 | Publicado en 03 Setiembre 2021

\section{RESUMEN}

El problema se ha identificado en el ejercicio de la asesoría financiera para las medianas empresas comerciales de Lima Metropolitana, en buena cuenta dichas empresas no vienen recibiendo la adecuada consejería para sus inversiones, deudas, liquidez, gestión, solvencia y rentabilidad de las empresas mencionadas, lo cual se expresa en la siguiente pregunta general: ¿De qué manera la formación y desarrollo profesional del Contador Público podrá influir en la eficiente asesoría financiera? La expresión del problema requiere la presentación de ese elemento metodológico que permite expresar la solución del mismo; siendo que por su parte se tiene a la hipótesis general: La formación y desarrollo profesional del Contador Público influye en grado significativo en la eficiente asesoría financiera; mediante la formación humanística, ética y moral; formación académica del Contador Público; capacitación profesional; entrenamiento profesional; $\mathrm{y}, \quad$ perfeccionamiento profesional del Contador Público. En el mismo contesto se presenta el objetivo general del trabajo: Determinar la manera como la formación y desarrollo profesional del Contador Público podrá influir en la eficiente asesoría financiera. En promedio el $90 \%$ de los encuestados considera que la formación y desarrollo profesional del Contador Público influye en grado significativo en la eficiente asesoría financiera.

Palabras clave: Formación y desarrollo profesional del Contador Público; asesoría financiera y medianas empresas de Lima Metropolitana.

\section{ABSTRACT}

The problem has been identified in the exercise of financial advice for mediumsized commercial companies in Metropolitan Lima, in good account these companies have not been receiving adequate advice for their investments, debts, liquidity, management, solvency and profitability of the companies mentioned, which is expressed in the following general question: How can the training and professional development of the Public Accountant influence the efficient financial advice? The expression of the problem requires the presentation of that methodological element that allows expressing its solution; being that for its part we have the general hypothesis: The training and professional development of the Public Accountant influences to a significant degree the efficient financial advice; through humanistic, ethical and moral training; academic training of the Public Accountant; professional training; Professional training; and, professional improvement of the Public Accountant. In the same answer, the general objective of the work is presented: To determine the way in which the training and professional development of the Public Accountant can influence the efficient financial advice. On average, $90 \%$ of those surveyed consider that the training and professional development of the Public Accountant has a significant influence on efficient financial advice.

Keywords: Training and professional development of the Public Accountant; financial advice and medium-sized companies in Metropolitan Lima.

\section{RESUMO}

O problema foi identificado no exercício de assessoria financeira para empresas comerciais de médio porte na região metropolitana de Lima, em boa conta essas empresas não têm recebido assessoria adequada para seus investimentos, dívidas, liquidez, gestão, solvência e rentabilidade das empresas mencionadas, que se expressa na seguinte questão geral: Como a formação e o desenvolvimento profissional do Contador Público influenciam a assessoria financeira eficiente? A expressão do problema requer a apresentação daquele elemento metodológico que permita expressar a sua solução; sendo que por seu lado temos a hipótese geral: A formação $\mathrm{e}$ desenvolvimento profissional do Contabilista Público influencia de forma significativa a assessoria financeira eficiente; pela formação humanística, ética e moral; formação académica do Contabilista Público; treinamento profissional; Treinamento profissional; e, aperfeiçoamento profissional do Contador Público. Na mesma resposta, apresenta-se o objetivo geral do trabalho: Determinar de que forma a formação e o desenvolvimento profissional do Contabilista Público podem influenciar a assessoria financeira eficiente. Em média, 90\% dos inquiridos consideram que a formação e desenvolvimento profissional do Contabilista Público tem uma influência significativa numa assessoria financeira eficiente.

Palavras-chave: Formação e desenvolvimento profissional do Contador Público; assessoria financeira e empresas de médio porte na região metropolitana de Lima. 


\section{Introducción}

Estado actual del problema: El problema de la asesoría financiera incluye la formulación de información financiera constituida en los estados financieros de acuerdo con las Normas Internacionales de Información Financiera; analizar la información financiera para disponer de datos financieros y contables; interpretar la información financiera para poder dar las recomendaciones para los usuarios; dar pautas para la toma de decisiones financieras sobre inversiones, deudas, rentabilidad y riesgos; como hacer que la asesoría financiera ayude en la mejora continua financiera como mejora continua general de las empresas. Todo lo cual se convierte en problema cuando se confunde capital de trabajo bruto con bienes de capital; deudas internas con deudas externas; como rentabilidad con liquidez; como liquidez con solvencia; valor actual neto con rentabilidad real; etc.

De otro lado se tiene que el problema del ejercicio de la asesoría financiera para las empresas se da en varias partes del mundo tal como se indica a continuación. Al respecto, Martínez (2016) considera que es necesario disponer de un modelo de optimización de las necesidades operativas de fondos. En cambio Gonzáles (2018) considera que la gestión financiera es la puerta para el acceso a financiamiento de las pymes del sector comercio en la ciudad de Bogotá.

Localmente, el problema se ha identificado en el ejercicio de la asesoría financiera para las medianas empresas comerciales de Lima Metropolitana, esto se concreta en la deficiente formulación de información financiera; falta de análisis de la información financiera, en otros casos deficiente análisis de la información financiera y falta de aprovechamiento de la información financiera. También se ha determinado la deficiente interpretación de información financiera en relación a la estructura de inversiones, deudas como ventas, costos, gastos y resultados. Asimismo, se ha determinado una 
deficiente toma de decisiones financieras, porque no se tiene las recomendaciones más adecuadas para dichas decisiones. Todo lo anterior se culmina en la falta de mejora continua financiera, al no haber creatividad ni innovación en el uso de la información financiera para cumplir las metas, objetivos y generación de valor financiero para las medianas empresas de Lima Metropolitana. Del 100\% de Contadores Públicos de Lima Metropolitana, según información del Colegio de Contadores Públicos de Lima; solo un $12 \%$ se dedica a la asesoría financiera; la mayor parte está en tributario y auditoría. La deficiente asesoría financiera conlleva a tener deficiente capital de trabajo para el funcionamiento operacional de las medianas empresas comerciales de Lima Metropolitana; como deficiente bienes de capital de las empresas indicadas. Por otra parte, la deficiente asesoría financiera, también conlleva a no tener una adecuada estructura de deudas, propia y con terceros, lo que no da el mejor apalancamiento para las medianas empresas comerciales de Lima Metropolitana.

Problema general: ¿De qué manera la formación y desarrollo profesional del Contador Público podrá influir en la eficiente asesoría financiera para las medianas empresas de Lima Metropolitana?

Problemas específicos: ¿De qué forma la formación humanista, ética y moral del Contador Público podrá facilitar la eficiente asesoría financiera para las medianas empresas de Lima Metropolitana? ¿De qué modo la formación académica del Contador Público podrá incidir en la eficiente asesoría financiera para las medianas empresas de Lima Metropolitana? ¿De qué manera la capacitación profesional del Contador Público podrá influir en la eficiente asesoría financiera para las medianas empresas de Lima Metropolitana? ¿De qué forma el entrenamiento profesional del Contador Público podrá incidir en la eficiente asesoría financiera para las medianas empresas de Lima Metropolitana? ¿De qué modo el perfeccionamiento profesional del Contador Público 
podrá influir en la eficiente asesoría financiera para las medianas empresas de Lima Metropolitana?

Antecedentes nacionales: Ayay (2016) concluye que existe relación significativa entre la formación profesional y el desempeño laboral, en los docentes de la Universidad Nacional Toribio Rodríguez de Mendoza de Amazonas. Entretanto Sumari (2016) concluye que los factores que determinan a la educación financiera son: capacidad de ahorro, hábitos de compra y el uso y conocimiento de productos financieros. Por su parte Recavarren (2014) considera que la exitosa adopción de los IFRS en 2005 por la Unión Europea impulsó un cambio radical en las actitudes hacia estándares contables comunes para la presentación de reportes financieros estandarizados. En cambio para Patricio, (2017) el Contador Público debe tener una sólida formación en Normas Internacionales de Información Financiera, para formular, analizar, interpretar y recomendar para la toma de decisiones financieras de las empresas. Más claramente Hernández (2016) determina que la contabilidad financiera es relevante; sin embargo para efectos de toma de decisiones, los directivos deberían complementarse con información económica, administrativa, financiera, estadística, legal, laboral, tributaria y otras fuentes de tal modo que se tomen decisiones bien informadas. De otro lado Matia (2016) se centra en un análisis histórico-legal de los procesos de formación profesional y formación para el empleo en España. En cambio Martínez (2018) señala que los docentes ven necesarios el aprendizaje permanente, el asesoramiento y la orientación del alumnado. Por su parte Mina (2015) procura plantear y fundamentar la necesidad de priorizar a la ética como componente esencial en la formación del profesional de la ciencia contable. Para Criollo (2019) es imprescindible tener amplia información para la toma de decisiones adecuadas. Para Bertel \& Carbono (2019) es muy importante que los gestores tengan la información financiera y económica de las empresas para que tomen decisiones eficientes. 


\section{Bases teóricas:}

Formación y desarrollo profesional del contador público: Huaynalaya (2013); interpreta a Frigo (2012); y señala que la formación y desarrollo profesional es toda actividad realizada en una empresa, respondiendo a sus necesidades, que busca mejorar. Para Salas (2018) los retos de la educación superior para el Siglo XXI plantean la necesidad de un nuevo proceso educativo, fundamentado en los principios de excelencia, calidad y pertinencia. En opinión de Vezub (2017) la mayoría de los programas de mejora de los sistemas educativos en América Latina deben fortalecer las competencias y la profesionalidad. Para Rodríguez, Cisterna \& Gallegos (2011) el escenario mundial ha mostrado cambios vertiginosos en la educación. En la opinión de Dextre (2013) la educación superior debe ser capaz de atender las necesidades de un mercado laboral que tiende a ser cada vez más moderno y competitivo.

Asesoría financiera empresarial: Según Gitman (2014) la asesoría financiera empresarial es el conjunto de sugerencias sobre inversiones, deudas, rentabilidad y riesgos. Para Weston (2014) en la administración financiera de las empresas, un tema clave es la asesoría financiera empresarial. Por su parte Bravo, Lambretón \& Márquez (2014) consideran que las decisiones financieras empresariales serán mejores en la medida que se cuente con una adecuada asesoría financiera empresarial. En la teoría de Van Horne (2014) la administración financiera empresarial se refiere a la forma como se planifica, organiza, dirige y controla las finanzas. Para Duran (2014) la administración financiera empresarial es una combinación de las finanzas y la administración a favor de las empresas. Según Santandreu \& Santandreu (2018) la administración financiera de las empresas se lleva a cabo en forma más eficiente y efectiva mediante una adecuada asesoría financiera profesional. En cambio para Quispe (2015) la asesoría financiera empresarial es la clave para el buen manejo de la liquidez, gestión, solvencia y 
rentabilidad de las empresas. De otro lado, Ross (2014) estima que la asesoría financiera empresarial es la clave para la reducción del riesgo y el incremento de la rentabilidad empresarial.

\section{Marco conceptual:}

Formación humanista, ética y moral: Según Frigo (2012) la formación humanista proporciona al profesional los elementos cognoscitivos indispensables para comprender mejor el mundo.

Formación académica: Según Frigo (2012) la formación académica es un conjunto de conocimientos adquiridos en el marco de los estudios de la carrera profesional, los cuales son la columna vertebral que ayudará a consolidar las competencias posteriormente cuando se desarrolle en el campo profesional.

Capacitación Profesional: Según Frigo (2012) la capacitación profesional es el procedimiento organizado por el cual los profesionales comprenden determinados temas profesionales.

Entrenamiento profesional: Para Frigo (2012) el entrenamiento profesional está referido a la praxis profesional en las áreas de la especialidad del profesional.

Perfeccionamiento profesional: Frigo (2012) considera que el perfeccionamiento profesional puede relacionarse con los estudios de posgrado y diplomados especializados. Formulación de información financiera: Según Van Horne (2014) la formulación de información financiera se refiere a la preparación de los estados financieros de una empresa; es decir la formulación del producto final de la contabilidad financiera.

Análisis de información financiera: Van Horne (2014) considera que el análisis de la información financiera consiste en estudiar la información contenida en los estados financieros. 
Interpretación de información financiera: Según Van Horne (2014) la interpretación de información financiera deviene del entendimiento de la información financiera.

Toma de decisiones financieras: Según de Van Horne (2014) la toma de decisiones financieras es la elección entre dos o más alternativas para mejorar la situación empresarial.

Mejora continua financiera: Según Van Horne (2014) la mejora continua financiera se refiere a la creatividad e innovación financiera que deben tener permanentemente las empresas. La mejora continua financiera es la puerta para la buena gestión, para alcanzar competitividad y generar valor agregado en las empresas.

Medianas Empresas Comerciales de Lima Metropolitana: Según Rodríguez (2018) con la promulgación de la Ley $\mathrm{N}^{\mathrm{o}} 30056$, de alguna manera recién aparece la indicación de que se entiende por mediana empresa, antes de esta norma solo se deducía cuáles podrían ser medianas empresas, en cambio esta norma lo indica taxativamente. Para Rodríguez (2018) las medianas empresas comerciales de Lima Metropolitana son unidades económicas constituidas por una persona jurídica, bajo cualquier forma de organización o gestión empresarial contemplada en la legislación vigente, que tiene como objeto la comercialización de bienes. Por su parte Argibay (2018) sostiene que las medianas empresas desarrollan sus actividades y llevan a cabo una serie de transacciones como compras, ventas, cobros, pagos por mencionar lo elemental; todas esas transacciones con consideradas por la contabilidad financiera y de cuyo tratamiento se obtienen los estados financieros que sirven de base para la toma de decisiones. Analizando a Pérez (2018), el gerente o administrador de las medianas empresas comerciales de Lima Metropolitana, tienen el deber de crear, y luego dirigir, toda una serie de relaciones entre su empresa y sus trabajadores, proveedores, bancos y clientes. Para Ortega (2018) la sociedad puede desarrollar actividades lícitas, formales y legales. 
Justificación: Este trabajo está plenamente justificado en el sentido que frente al problema de la deficiente asesoría financiera para las medianas empresas de Lima Metropolitana; se presenta como solución a la formación y desarrollo profesional del Contador Público; por cuanto este dotará de los atributos y valores, como las estrategias, tácticas y acciones para tener la mayor información posible, procesarla y entregarla a los responsables de la gestión de las empresas que son motivo de estudio.

Objetivo general: De la manera como la formación y desarrollo profesional del Contador Público podrá influir en la eficiente asesoría financiera para las medianas empresas de Lima Metropolitana.

Objetivos específicos: 1) Establecer la forma como la formación humanista, ética y moral del Contador Público podrá facilitar la eficiente asesoría financiera para las medianas empresas de Lima Metropolitana. 2) Determinar el modo como la formación académica del Contador Público podrá incidir en la eficiente asesoría financiera para las medianas empresas de Lima Metropolitana. 3) Establecer la manera como la capacitación profesional del Contador Público podrá influir en la eficiente asesoría financiera para las medianas empresas de Lima Metropolitana. 4) Determinar la forma como el entrenamiento profesional del Contador Público podrá incidir en la eficiente asesoría financiera para las medianas empresas de Lima Metropolitana. 5) Establecer el modo como el perfeccionamiento profesional del Contador Público podrá influir en la eficiente asesoría financiera para las medianas empresas de Lima Metropolitana.

Hipótesis general: La formación y desarrollo profesional del Contador Público influye en grado significativo en la eficiente asesoría financiera para las medianas empresas de Lima Metropolitana; mediante la formación humanística, ética y moral; formación académica del Contador Público; capacitación profesional; entrenamiento profesional; y, perfeccionamiento profesional del Contador Público. 
Hipótesis específicas: 1) La formación humanista, ética y moral del Contador Público facilita en grado significativo en la eficiente asesoría financiera para las medianas empresas de Lima Metropolitana; mediante la formación en calidad humana, valores y comportamiento cabal del Contador Público. 2) La formación académica del Contador Público incide en grado significativo en la eficiente asesoría financiera para las medianas empresas de Lima Metropolitana; mediante la formación en diferentes áreas del saber relacionadas con la profesión contable tales como contabilidad, auditoría, costos, finanzas, tributación, sistemas contables e investigación contable. 3) La capacitación profesional del Contador Público influye en grado significativo en la eficiente asesoría financiera para las medianas empresas de Lima Metropolitana; mediante el adiestramiento en las diferentes áreas de la profesión contable tales como contabilidad, auditoría, costos, finanzas, tributación, sistemas contables e investigación contable. 4) El entrenamiento profesional del Contador Público incide en grado significativo en la eficiente asesoría financiera para las medianas empresas de Lima Metropolitana; mediante la práctica intensiva en las diferentes áreas de la profesión contable tales como contabilidad, auditoría, costos, finanzas, tributación, sistemas contables e investigación contable. 5) El perfeccionamiento profesional del Contador Público influye en grado significativo en la eficiente asesoría financiera para las medianas empresas de Lima Metropolitana; mediante la mejora continua profesional en las áreas de la profesión contable tales como contabilidad, auditoría, costos, finanzas, tributación, sistemas contables e investigación contable.

\section{Materiales y métodos}

Tipo de investigación: La formación y desarrollo profesional del Contador Público en el ejercicio de la asesoría financiera para las medianas empresas comerciales de Lima 
Metropolitana es una investigación de tipo aplicada; por cuanto se propone aplicar dicho trabajo tanto en los Contadores Públicos como en las empresas indicadas.

Diseño de la investigación. El diseño que se ha tenido en cuenta en este trabajo ha sido el no experimental.

Población: Se tomó en cuenta una población que estuvo conformada por 10,000 personas relacionadas.

Muestra: La muestra que estuvo conformada por 370 personas relacionadas.

Área de estudio: La formación y desarrollo profesional del Contador Público; ejercicio de la asesoría financiera y medianas empresas comerciales de Lima Metropolitana

Procedimientos estadísticos: Estadísticamente se ha aplicado la correlación para determinar el grado de asociación de las variables del trabajo; también el grado de significancia para haber aceptado la hipótesis alternativa y rechazar la hipótesis nula.

Aspectos éticos: Zavala \& Alfaro (2018) hacen una evaluación crítica de los aspectos éticos de la investigación en base a su experiencia como docentes universitarios y como miembros de Comités de Ética en Investigación. Invitan a la discusión de temas que consideran polémicos. Comienzan mencionando el rol regulador de las Normas Éticas Internaciones y Locales y de los Comités de Ética en Investigación.

\section{Resultados}

Respecto de los resultados se tiene que el $90 \%$ de los encuestados acepta que la formación y desarrollo profesional del Contador Público debe tener sentido humanista, ético, moral, académico y tecnológico para una efíciente asesoría. El 90\% de los encuestados acepta que la formación humanista es fundamental para que el Contador Público facilite asesoría financiara. El 88\% de los encuestados acepta que la formación ética y moral es fundamental para que el Contador Público intervenga en la asesoría financiera. El 90\% de los encuestados acepta que la formación académica del Contador Público tiene que ser 
científica y tecnológica para que intervenga en la asesoría financiera. El 88\% de los encuestados acepta que la formación académica debe incluir la investigación en todos sus aspectos para formar al Contador Público y facilitar la asesoría financiera. El 90\% de los encuestados acepta que la capacitación profesional es clave para que el Contador Público esté en condiciones de facilitar asesoría financiera. E1 88\% de los encuestados acepta que la capacitación en finanzas, contabilidad, tributación, costos y auditoría financiera es de gran ayuda para que el Contador Público intervenga en la asesoría financiera. El 88\% de los encuestados acepta que el entrenamiento profesional del Contador Público facilita el papel de asesoría financiera. El $90 \%$ de los encuestados acepta que el entrenamiento profesional debe ser eventual y/o permanente para hacer del Contador Público un profesional de polendas en la asesoría financiera. El $88 \%$ de los encuestados acepta que el perfeccionamiento profesional es de gran ayuda para que el Contador Público pueda llevar a cabo el trabajo de asesoría financiera. El 88\% de los encuestados acepta que la falta de perfeccionamiento profesional es una limitante que debe superar el Contador Público para llevar a cabo la asesoría financiera.

E1 90\% de los encuestados acepta que la asesoría financiera es un campo de mucho futuro en el trabajo del Contador Público. El 90\% de los encuestados acepta que la formulación de información financiera es fundamental para conocer y comprender la situación empresarial. El 94\% de los encuestados acepta que el Contador Público debe formular el estado de situación financiera, estado de resultados, estado de cambios en el patrimonio neto y estado de flujos de efectivo para comprender la situación. El $78 \%$ de los encuestados acepta que el análisis de la información financiera permite comprender la estructura de las inversiones, deudas, ingresos, costos y gastos. El 94\% de los encuestados acepta que el análisis de información financiera permite obtener indicadores o ratios de liquidez, gestión, solvencia y rentabilidad. El 94\% de los encuestados acepta que la 
interpretación de información financiera es fundamental para que el Contador Público pueda asesorar financieramente las medianas empresas de Lima Metropolitana. El 94\% de los encuestados acepta que la interpretación ayuda a formarse una idea de la salud empresarial. E1 94\% de los encuestados acepta que la toma de decisiones financieras debe incluir decisiones de financiamiento e inversiones. E1 89\% de los encuestados acepta que la toma de decisiones financieras debe influir decisiones de rentabilidad y sobre riesgos. El 94\% de los encuestados acepta que como consecuencia de la eficiente asesoría financiera se podrá lograr la mejora continua. El 94\% de los encuestados acepta que la creatividad, innovación, reducción de costos y reducción de gastos es parte de la mejora continua financiera.

\section{Contrastación de las hipótesis:}

Contrastación de la hipótesis general: La correlación o grado de asociación entre las variables es igual a $90.42 \%$. Además, se tiene un valor de significancia igual a $4.08 \%$, porcentaje que menor al margen de error propuesto del $5.00 \%$. Se concluye que, la formación y desarrollo profesional del Contador Público influye en grado significativo en la eficiente asesoría financiera.

Contrastación de la hipótesis específica 1: La correlación o grado de asociación entre las variables es igual a $90.09 \%$ y el valor de significancia, igual a $4.11 \%$. Se concluye que, la formación humanista, ética y moral del Contador Público facilita en grado significativo en la eficiente asesoría financiera.

Contrastación de la hipótesis específica 2: La correlación o grado de asociación entre las variables es igual a $90.07 \%$ y un valor de significancia, igual a $4.36 \%$. Se concluye que la formación académica del Contador Público incide en grado significativo en la eficiente asesoría financiera. 
Contrastación de la hipótesis específica 3: La correlación o grado de asociación entre las variables es igual a 90.02 y el valor de significancia igual a $3.49 \%$. Se concluye que, la capacitación profesional del Contador Público influye en grado significativo en la eficiente asesoría financiera.

Contrastación de la hipótesis específica 4: La correlación o grado de asociación entre las variables es igual a 90.37\% y el valor de significancia igual a $4.88 \%$. Se concluye que, el entrenamiento profesional del Contador Público incide en grado significativo en la eficiente asesoría financiera.

Contrastación de la hipótesis específica 5: La correlación o grado de asociación entre las variables es igual a $90.24 \%$. Además se tiene un valor de significancia igual a $4.55 \%$. Se concluye que el perfeccionamiento profesional del Contador Público influye en grado significativo en la eficiente asesoría financiera.

\section{Discusión}

Discusión del resultado de la variable independiente: E1 90\% de los encuestados acepta que la formación y desarrollo profesional del Contador Público debe tener sentido humanista, ético, moral, académico y tecnológico para una eficiente asesoría de las medianas empresas de Lima Metropolitana. Este resultado es similar al 92\% presentado, aunque en otra dimensión espacial y temporal, por Matia, V. (2016). Formación profesional y formación para el empleo en España: del aprendizaje de tareas al desarrollo de competencias. (Tesis Doctoral). Universidad de Valladolid. Valladolid, España. Ambos resultados son altos y favorecen el modelo de investigación desarrollado. La formación y desarrollo profesional del Contador Público ha sido fundamental todo el tiempo, pero lo es ahora más que otros tiempos; porque existe una elevada competencia profesional, que a diferencia de antes ahora requiere acreditaciones reacreditaciones profesionales; $\mathrm{y}$, además grados académicos; y desde luego la experiencia profesional del 
caso, incluido la aplicación de idiomas y tecnología de la información y comunicación (TIC).

Discusión del resultado de la variable dependiente: El 90\% de los encuestados acepta que la asesoría financiera es un campo de mucho futuro en el trabajo del Contador Público de las medianas empresas de Lima Metropolitana. Este resultado es similar al 91\% presentado, aunque en otra dimensión espacial y temporal, por Criollo, P. (2019). Implementación de una asesoría financiera, tributaria y contable con servicio personalizado en la Ciudad de Otavalo Provincia de Imbabura. (Tesis de Maestría). Universidad Técnica Del Norte. Ibarra, Ecuador. Ambos resultados son altos y favorecen el modelo de investigación desarrollado. Para ejercer la asesoría financiera, el Contador Público, aprueba una curricula académica del pregrado, con todo ese arsenal de conocimientos egresa y tiene que continuar perfeccionandose a través de diplomados. También tiene que estudiar posgrado en especialidades relacionadas.

\section{Conclusiones}

Conclusión general: Se ha determinado la manera como la formación y desarrollo profesional del Contador Público podrá influir en la eficiente asesoría financiera; mediante la formación humanista, ética y moral; formación académica; capacitación profesional; entrenamiento profesional y perfeccionamiento profesional.

Conclusiones específicas: 1) Se ha establecido la forma como la formación humanista, ética y moral del Contador Público podrá facilitar la eficiente asesoría financiera; mediante la conducta adecuada y la aplicación de valores con los colegas como con los clientes a los cuales se les facilita la asesoría financiera. 2) Se ha determinado el modo como la formación académica del Contador Público podrá incidir en la eficiente asesoría financiera; mediante la aprobación de las asignaturas de la curricula académica relacionadas con las finanzas empresariales, la misma que debe incluir los aspectos 
teóricos respectivos como la práctica correspondiente, de tal modo que se tenga todos los elementos para un buen asesoramiento. 3) Se ha establecido la manera como la capacitación profesional del Contador Público podrá influir en la eficiente asesoría financiera; mediante el llevado de diplomados de finanzas; cursos especializados de finanzas, conferencias de finanzas, seminarios de finanzas, webinar de finanzas y otros eventos que permitan estar actualizado en los aspectos financieros de las empresas. 4) Se ha determinado la forma como el entrenamiento profesional del Contador Público podrá incidir en la eficiente asesoría financiera; mediante la práctica en simuladores de finanzas como la obtención de experiencias sobre inversiones, financiamiento, rentabilidad, riesgo; liquidez, gestión, solvencia y otros aspectos de las finanzas empresariales. 5) Se ha establecido el modo como el perfeccionamiento profesional del Contador Público podrá influir en la eficiente asesoría financiera; mediante el llevado maestrías en finanzas, proyectos, banca, contabilidad, administración y otras relacionadas; como el llevado de doctorados en finanzas, contabilidad, administración, economía y otros similares de tal modo que se tenga el mayor nivel académico que garantice una asesoría financiera de calidad.

\section{Recomendaciones}

Recomendación general: Se recomienda a los propietarios, socios y accionistas tener en cuenta que la formación y desarrollo profesional del Contador Público influye en grado significativo en la eficiente asesoría financiera; mediante la formación humanística, ética y moral; formación académica del Contador Público; capacitación profesional; entrenamiento profesional; y, perfeccionamiento profesional del Contador Público; por tanto, deberían apoyar la formación y desarrollo profesional.

Recomendaciones específicas: 1) Se recomienda a los propietarios, socios y accionistas tener en cuenta que la formación humanista, ética y moral del Contador Público facilita 
en grado significativo en la eficiente asesoría financiera; mediante la formación en calidad humana, valores y comportamiento cabal del Contador Público; por tanto, deberían apoyar y exigir que se cumpla dicho tipo de formación. 2) Se recomienda a los propietarios, socios y accionistas tener en cuenta que la formación académica del Contador Público incide en grado significativo en la eficiente asesoría financiera; mediante la formación en diferentes áreas del saber relacionadas con la profesión contable tales como contabilidad, auditoría, costos, finanzas, tributación, sistemas contables e investigación contable; por tanto, deberían coordinar con las universidades para actualizar permanentemente el perfil profesional. 3) Se recomienda a los propietarios, socios y accionistas tener en cuenta que la capacitación profesional del Contador Público influye en grado significativo en la eficiente asesoría financiera; mediante el adiestramiento en las diferentes áreas de la profesión contable tales como contabilidad, auditoría, costos, finanzas, tributación, sistemas contables e investigación contable; por tanto, deberían apoyar. 4) Se recomienda a los propietarios, socios y accionistas tener en cuenta que el entrenamiento profesional del Contador Público incide en grado significativo en la eficiente asesoría financiera; mediante la práctica intensiva en las diferentes áreas de la profesión contable tales como contabilidad, auditoría, costos, finanzas, tributación, sistemas contables e investigación contable; por tanto, deberían apoyar para tener dicho entrenamiento profesional. 5) Se recomienda a los propietarios, socios y accionistas tener en cuenta que el perfeccionamiento profesional del Contador Público influye en grado significativo en la eficiente asesoría financiera para las medianas empresas de Lima Metropolitana; mediante la mejora continua profesional en las áreas de la profesión contable.

\section{Referencias}

Argibay, M. (2018). Contabilidad financiera y administración. Editorial Ideas 
Ayay, G. (2016). Relación entre la formación profesional y el desempeño laboral en los docentes de la Universidad Nacional Toribio Rodríguez de Mendoza de Amazonas. [Tesis de Maestría, Universidad Nacional de Trujillo]. Repositorio institucional:

http://dspace.unitru.edu.pe/bitstream/handle/unitru/3962/tesis\%20maestr\%c3\% ada\%20-\%20guido\%20ayay\%20arista.pdf?sequence=1\&isallowed=y

Bertel, S. \& Carbono, I. (2019). Creación de una empresa de asesoría financiera contable tributaria y de sistemas: Asesorías y auditorias empresariales "Saber" SA. [Tesis de maestría, Universidad de Bogotá]. Repositorio institucional: https://expeditiorepositorio.utadeo.edu.co/bitstream/handle/20.500.12010/1693 /t168.pdf?sequence $=1 \&$ isallowed $=y$

Bravo, M., Lambretón, V. \& Márquez, H. (2014). Introducción a las Finanzas. Editorial Pearson.

Criollo, P. (2019). Implementación de una asesoría financiera, tributaria y contable con servicio personalizado en la Ciudad de Otavalo Provincia de Imbabura. [Tesis de Maestría, Universidad Técnica del Norte]. Repositorio institucional: http://repositorio.utn.edu.ec/bitstream/123456789/1362/1/tesis\%20completa.pd f

Dextre, J. (2013). Los retos de la formación por competencias del Contador Público. [Artículo científico]. Contabilidad y Negocios, vol. 8, núm. 16, 2013, pp. 35-47. Recuperada de: https://www.redalyc.org/pdf/2816/281630133004.pdf

Durán, J. (2014). Decisiones financieras empresariales. Editorial Pirámide.

Frigo, E. (2012). Capacitación y beneficio para la organización. Editorial Norma.

Gitman, J. (2014). Fundamentos de Administración Financiera. Editorial Harper \& Row Latinoamericana. 
Gonzáles, S. (2018). La gestión financiera y el acceso a financiamiento de las pymes del sector comercio en la ciudad de Bogotá. [Tesis doctoral, Universidad Nacional de Colombia].

Repositorio institucional: http://www.bdigital.unal.edu.co/49025/1/tesis\%20la\%20gesti\%c3\%b3n\%20fin anciera $\% 20 \mathrm{y} \% 20 \mathrm{el} \% 20$ acceso $\% 20 \mathrm{a} \% 20$ financiamiento $\% 20 \mathrm{de} \% 201 \mathrm{las} \% 20$ pym es $\% 20 \mathrm{del} \% 20$ sector $\% 20$ comercio $\% 20$ en $\% 201 \mathrm{a} \% 20$ ciudad $\% 20 \mathrm{de} \% 20$ bogot $\%$ c $3 \%$ al.pdf

Hernández, D. (2016). La contabilidad financiera y la toma de decisiones en las Grandes Empresas Comerciales de Lima Metropolitana. [Tesis doctoral, Universidad Inca Garcilaso de la Vega]. Repositorio institucional: http://repositorio.uigv.edu.pe/bitstream/handle/20.500.11818/1194/t doctorado \%20en\%20administracion_40878290_hernandez_celis_domingo.pdf?sequence $=1 \&$ isallowed $=\mathrm{y}$

Huaynalaya, O. (2013). Capacitación, herramienta efectiva para la mejora continua de las Mutuales en el Perú. [Tesis doctoral, Universidad Nacional Federico Villarreal]. Repositorio institucional: Recuperado de: http://www.monografias.com/trabajos85/gerencia-efectiva-mejora-continuamutuales-peru/gerencia-efectiva-mejora-continua-mutuales-peru.shtml

Martínez, J. (2016). Modelo de gestión financiera basado en la optimización de las necesidades operativas de fondos: el caso de las empresas farmacéuticas en España. [Tesis Doctoral, Universidad Complutense de Madrid]. Repositorio institucional: https://eprints.ucm.es/40638/1/t38190.pdf

Martínez, M. (2018). Análisis y valoración de los ciclos de formación profesional básica como medida de atención a la diversidad a través del alumnado y del profesorado. [Tesis Doctoral, Universidad de Córdova]. Repositorio 
institucional:

https://helvia.uco.es/bitstream/handle/10396/16402/2018000001752.pdf?seque nce $=1 \&$ isAllowed $=\mathrm{y}$

Matia, V. (2016). Formación profesional y formación para el empleo en España: del aprendizaje de tareas al desarrollo de competencias. [Tesis Doctoral, Universidad de Valladolid]. Repositorio institucional: https://uvadoc.uva.es/bitstream/handle/10324/22300/tesis1223170217.pdf;jsessionid=99c02411aef70099763207ff41905602? sequence $=1$

Mina, S. (2015). La ética como componente transversal del plan de estudios para la formación del Contador Público colombiano. [Tesis Doctoral, Universidad de Santo Tomás].

Repositorio institucional: https://repository.usta.edu.co/bitstream/handle/11634/277/la\%20etica\%20como $\% 20$ componente $\% 20$ trasversal\%20del $\% 20$ plan $\% 20 \mathrm{de} \% 20$ estudios.pdf?sequen ce $=1 \&$ isallowed $=y$

Ortega, P. (2013). Organización de las empresas. Editorial San Marcos

Patricio, S. (2017). Las normas internacionales de información financiera y la toma de decisiones financieras en las Plantas Envasadoras de Gas del Perú, propuesta actual. [Tesis doctoral, Universidad Nacional Federico Villarreal]. Repositorio institucional: https://repositorio.unfv.edu.pe/

Pérez, E. (2018). Organización y Administración de empresas comerciales en el Perú. Editorial San Marcos

Quispe, U. (2015). Administración Financiera. Editorial San Marcos

Recavarren, F. (2014). Impacto de las normas internacionales de información financiera en el desarrollo de las Grandes Empresas Familiares del Perú. [Tesis doctoral, Universidad de San Martín de Porres] Repositorio institucional: 
http://www.repositorioacademico.usmp.edu.pe/bitstream/usmp/1121/1/recavarr en_r.pdf

Rodríguez, E., Cisterna, F. \& Gallegos, C. (2018). El sistema de prácticas como elemento integrante de la formación profesional. México. [Artículo científico]. Recuperada de: $\quad$ http://www.scielo.org.mx/scielo.php?pid=s0185$27602011000300004 \&$ script $=$ sci_arttext\&tlng=pt

Rodríguez, L. (2018). Planificación y dirección de las empresas comerciales. Editorial Continental SA.

Ross, S. (2014). Administración financiera.: Mc Graw Hill.

Salas, R. (2018), La calidad en el desarrollo profesional: avances y desafios. [artículo científico]. Recuperado de: http://scielo.sld.cu/scielo.php?script=sci_arttext\&pid=S086421412000000200003

Santandreu, E. \& Santandreu, P. (2018). Manual de Finanzas. Gestión 2000.

Servicio Nacional de Capacitación y Empleo (03 de mayo del 2017). Glosario técnico de términos de capacitación y empleo. Recuperado de: http://empresas.sence.cl/documentos/estudios/glosario_tecnico.pdf

Sumari, H. (2016). Factores determinantes de la educación financiera en personas adultas de la urbanización San Santiago de la ciudad de Juliaca, periodo 2015. [Tesis de Título Profesional, Universidad Peruana Unión]. Repositorio institucional:

http://repositorio.upeu.edu.pe:8080/bitstream/handle/upeu/448/judith_tesis_bac hiller_2016.pdf?sequence $=1 \&$ isallowed $=\mathrm{y}$

Van Horne, J. (2014). Administración financiera. Mc Graw Hill.

Van Horne, W. (2013). Fundamentos de Administración financiera. Mc Graw Hill 
Vezub, L. (2017). La formación y el desarrollo profesional docente frente a los nuevos desafios de la escolaridad. Recuperada de: https://www.redalyc.org/pdf/567/56711102.pdf

Weston, J. (2017). Finanzas. Mc Graw Hill

Zavala, S. \& Alfaro, J. (2018). Ética e investigación. [Artículo científico]. Recuperado de: https://www.scielosp.org/scielo.php?pid=s172646342011000400015\&script=sci_arttext\&tlng=pt 